\section{Commentary: Spontaneous myocardial infarctions and the vital choice: Bypass or stent}

\author{
Tohru Asai, MD, PhD
}

Coronary artery bypass grafting $(\mathrm{CABG})$ and percutaneous coronary intervention (PCI) have been compared in numerous randomized control trials (RCT) over 25 years. Both treat coronary artery disease (CAD) to relieve angina and myocardial ischemia but in very different ways. While flow-restricting coronary artery stenosis is locally dilated by PCI, a new inflow of blood supply through a bypass graft is constructed distal to the stenosis in CABG, an effect called "surgical collateralization." Particularly in severe CAD, that difference is believed to give rise to a difference in protection against subsequent spontaneous myocardial infarction (SMI). It has been suggested CABG offers an advantage over PCI by reducing the risk of SMI, but no investigation has yet proven this concept.

The surgical collateralization bypasses not only any present culprit occlusive lesions but also any future culprit lesions proximal to the anastomosis, as illustrated in Figure 1. It seems plausible that the blood supply from the bypass graft would protect the myocardial territory in the event of any later occlusion proximal to the anastomosis, whereas the local dilatation created by PCI would not.

SMI was recently brought to increased attention by publication of the 5-year outcomes of 2 RCTs for left main coronary artery disease, NOBLE (Nordic-Baltic-British left main revascularisation study) ${ }^{1}$ and EXCEL (Evaluation of XIENCE vs Coronary Artery Bypass Surgery for Effectiveness of Left Main Revascularization). ${ }^{2}$ Their conclusions were conflicting. Although the NOBLE trial demonstrated superiority of CABG over PCI, the EXCEL trial showed no difference. However, there was considerable controversy about the definition of myocardial infarction used in the

From the Department of Cardiovascular Surgery, Juntendo University, Tokyo, Japan. Disclosures: The author reported no conflicts of interest.

The Journal policy requires editors and reviewers to disclose conflicts of interest and to decline handling or reviewing manuscripts for which they may have a conflict of interest. The editors and reviewers of this article have no conflicts of interest.

Received for publication May 2, 2021; revisions received May 2, 2021; accepted for publication May 3, 2021; available ahead of print May 8, 2021.

Address for reprints: Tohru Asai, MD, PhD, Department of Cardiovascular Surgery, Juntendo University, Faculty of Medicine, 2-1-1, Hongo, Bunkyo-ku, Tokyo 1138421, Japan (E-mail: t.asai.jj@juntendo.ac.jp).

J Thorac Cardiovasc Surg 2023;165:670-1

$0022-5223 / \$ 36.00$

Copyright (c) 2021 by The American Association for Thoracic Surgery

https://doi.org/10.1016/j.jtcvs.2021.05.005

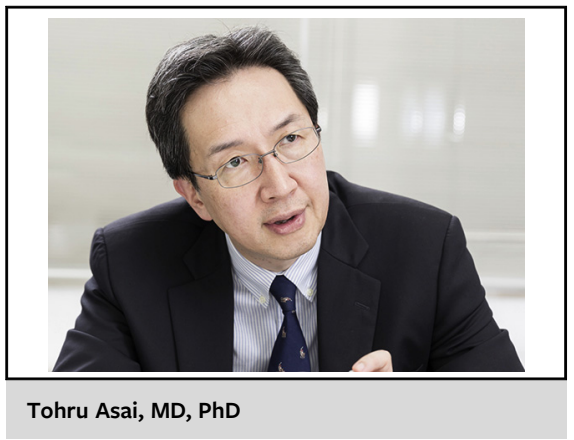

CENTRAL MESSAGE

The blood supply from the

bypass graft would protect the

myocardial territory in the event

of any later occlusion proximal to

the anastomosis, whereas the

local dilatation created by $\mathrm{PCl}$

would not.

EXCEL trial, since the investigators used a new protocol definition of MI that clearly penalized CABG surgery, particularly in periprocedural MI incidence. However, the EXCEL trial investigators ${ }^{3}$ conceded that the incidence of spontaneous MI after interventions was clearly lower in the surgical arm than in the PCI arm, as in the NOBLE trial.

In this issue of the Journal, Gaudino and colleagues ${ }^{4}$ present an elegant meta-analysis to demonstrate that a survival advantage is seen only in SMI after intervention. Their sophisticated study sought any correlation between all-cause mortality and SMI rather than simply showing a survival advantage with CABG. Among all the reports searched in databases comparing CABG and PCI with bare-metal or drug-eluting stent, 20 full-text RCT articles reporting allcause mortality and SMI were included in this metaanalysis. Seven trials $(35 \%)$ demonstrated significant reduction in SMI in the CABG arm, and significant reduction of all-cause mortality was found only in those trials. The findings support the hypothesis that the protecting mechanism against SMI creates a survival benefit with CABG.

This SMI-preventing effect of CABG would be most apparent in patients with rapidly progressing atherosclerosis. It is a unique advantage of CABG that should be kept in mind, not only by cardiologists and cardiac surgeons, but also by all patients with CAD. I am grateful to 

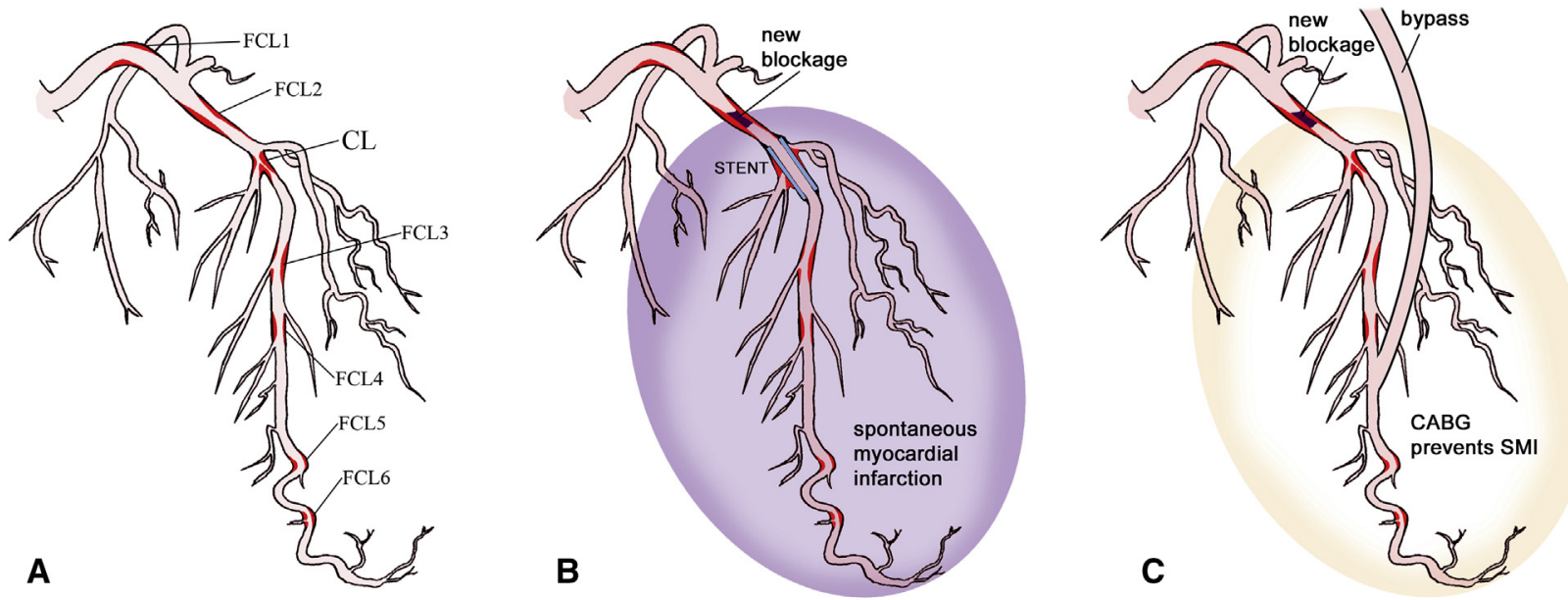

FIGURE 1. Benefit of surgical collateralization. A, Myocardial ischemia is caused by a flow-restricting "culprit" lesion $(C L)$, but other "future culprit" lesions $(F C L)$ also exist. B, When a new blockage occurs at another lesion later, spontaneous myocardial infarction (SMI) may develop despite the previously implanted stent. C, Alternatively, the blood supply from the bypass graft would prevent SMI. CABG, Coronary artery bypass grafting.

Gaudino and colleagues for presenting this succinct and significant finding to our readers.

\section{References}

1. Holm NR, Mäkikallio T, Lindsay MM, Spence MS, Erglis A, Menown IBA, et al. Percutaneous coronary angioplasty versus coronary artery bypass grafting in the treatment of unprotected left main stenosis: updated 5-year outcomes from the randomised, non-inferiority NOBLE trial. Lancet. 2020;395:191-9.
2. Stone GW, Kappetein AP, Sabik JF, Pocock SJ, Morice MC, Puskas J, et al. Fiveyear outcomes after PCI or CABG for left main coronary disease. $N$ Engl J Med. 2019;381:1820-30.

3. Stone GW, Serruys PW, Sabik JF. PCI or CABG for left main coronary artery disease. Reply. N Engl J Med. 2020;383:292-4.

4. Gaudino M, Di Franco A, Spadaccio C, Rahouma M, Robinson NB, Demetres M, et al. Difference in spontaneous myocardial infarction and mortality in percutaneous versus surgical revascularization trials: a systematic review and meta-analysis. J Thorac Cardiovasc Surg. 2023;165:662-9.e14. 